\title{
A random forest classifier predicts recurrence risk in patients with ovarian cancer
}

\author{
LI CHENG ${ }^{*}$, LIN LI ${ }^{*}$, LILING WANG, XIAOFANG LI, HUI XING and JINTING ZHOU \\ Department of Obstetrics and Gynecology, Xiangyang Central Hospital (Affiliated Hospital of \\ Hubei University of Arts and Science), Xiangyang, Hubei 441021, P.R. China
}

Received December 9, 2017; Accepted April 23, 2018

DOI: $10.3892 / \mathrm{mmr} .2018 .9300$

\begin{abstract}
Ovarian cancer (OC) is associated with a poor prognosis due to difficulties in early detection. The aims of the present study were to construct a recurrence risk prediction model and to reveal important OC genes or pathways. RNA sequencing data was obtained for 307 OC samples, and the corresponding clinical data were downloaded from The Cancer Genome Atlas database. Additionally, two validation datasets, GSE44104 (20 recurrent and 40 non-recurrent OC samples) and GSE49997 (204 OC samples), were obtained from the Gene Expression Omnibus database. Differentially expressed genes were screened using the differential expression via distance synthesis algorithm, followed by gene ontology enrichment analysis and weighted gene coexpression network analysis (WGCNA). Furthermore, subnetwork analysis was conducted for the protein-protein interaction (PPI) network using the BioNet package. Finally, a random forest classifier was constructed based on the subnetwork nodes, and its reliability was validated using the GSE44104 and GSE49997 validation datasets. A total of 44 upregulated and 117 downregulated genes were identified in the recurrent samples. Enrichment analysis indicated that cytochrome P450 family 17 subfamily A member 1 (CYP17A1) was associated with 'positive regulation of steroid hormone biosynthetic processes'. WGCNA identified turquoise and grey modules that were significantly correlated with status and prognosis. A significant PPI subnetwork containing 16 nodes was also identified, including: Transcription factor GATA-4; fibroblast growth factor 9; aromatase; $3 \beta$-hydroxysteroid dehydrogenase/ 85 -4-isomerase type 2 ; corticosteroid $11 \beta$-dehydrogenase
\end{abstract}

Correspondence to: Dr Jinting Zhou, Department of Obstetrics and Gynecology, Xiangyang Central Hospital (Affiliated Hospital of Hubei University of Arts and Science), 39 Jingzhou Street, Xiangcheng, Xiangyang, Hubei 441021, P.R. China

E-mail: xyzhoujinting@sina.com

*Contributed equally

Key words: ovarian cancer, random forest classifier, gene expression, weighted gene coexpression network analysis, subnetwork analysis isozyme 1; CYP17A1; pituitary homeobox 2; left-right determination factor 1; homeobox protein ARX; estrogen receptor $\beta$; steroidogenic factor 1; forkhead box protein L2; myocardin; steroidogenic acute regulatory protein mitochondrial; vesicular inhibitory amino acid transporter; and twist-related protein 1. A random forest classifier was constructed using the subnetwork nodes as feature genes, which exhibited a $92 \%$ true positive rate when classifying recurrent and non-recurrent OC samples. The classifying efficiency of the random forest classifier was validated using the two other independent datasets. Overall, 44 upregulated and 117 downregulated genes associated with OC recurrence were identified. Furthermore, the 16 subnetwork node genes that were identified may be important molecules in OC recurrence.

\section{Introduction}

Ovarian cancer (OC), which frequently occurs in postmenopausal women (1), is a cancer with no apparent symptoms until it reaches an advanced stage. The symptoms include bloating, abdominal swelling, pelvic pain and loss of appetite (2). The three most common OC subtypes are high-grade serous carcinomas, sex cord stromal tumors and germ cell tumors (3), which may metastasize to the peritoneum, liver, lungs or lymph nodes (4). OC is difficult to detect and metastasizes early in disease progression; therefore, patients with OC frequently have a poor prognosis (5). Globally, OC affects 1.2 million women and led to 161,100 mortalities in $2015(6,7)$. Thus, obtaining an improved understanding of OC progression and recurrence is of great importance for improving its prognosis.

Previously, genes affecting OC were identified, including p21-activated kinase 4 (Pak4), cyclin E1 (CCNE1), RNA binding motif protein 3 (RBM3), YY1 associated protein 1 $(Y A P)$ and prominin-1 (CD133). Pak4 overexpression has been reported to contribute to $\mathrm{OC}$ cell migration, invasion and proliferation, thus making it a promising prognostic indicator and therapeutic target (8). CCNE1 amplification has been demonstrated to markedly reduce disease-free survival and overall survival, thus indicating that CCNE1-targeted treatment may benefit patients with $\mathrm{OC}$ who have upregulated CCNE1 expression $(9,10)$. In epithelial ovarian cancer, $R B M 3$ expression has been associated with cisplatin sensitivity and correlated with a positive patient prognosis (11). Furthermore, 
$Y A P$ has been associated with cell growth and tumorigenesis, and its coexpression with TEA domain transcription factor 4 serves as a predictor of a poor outcome $(12,13)$. CD133 also serves as a predictor of poor OC patient survival, thus suggesting that it may serve as a biomarker of cancer stem cells during disease (14). However, the further identification of prognostic indicators and their potential uses is required.

In recent years, bioinformatics analysis of expression profile data has been gradually used to examine the pathogenesis of human diseases (15). It is known that the progress of a disease is usually mediated by multiple relevant genes and not by a single gene $(16,17)$. Therefore, the present study was designed to mine subnetwork features and build a model to assess OC recurrence risk. In the present study, OC expression profiles were downloaded from a public database, and differentially expressed genes (DEGs) were analyzed and functionally enriched. Following identification of a functional subnetwork, a random forest classifier was constructed and validated. It was considered that this constructed classifier may provide an improved approach for predicting the prognoses of patients with OC.

\section{Materials and methods}

Data source. RNA sequence data from 307 OC samples and their corresponding clinical data (including patient vital status and overall survival) were downloaded from The Cancer Genome Atlas (TCGA; https://cancergenome.nih.gov) database. Based on patient vital status, 180 recurrent samples and 72 disease-free samples were identified. Additionally, the GSE44104 dataset [including 20 recurrent and 40 non-recurrent OC samples; platform, (HG-U133_Plus_2) Affymetrix Human Genome U133 Plus 2.0 Array, Affymetrix; Thermo Fisher Scientific, Inc., Waltham, MA, USA] and the GSE49997 dataset (including 204 OC samples; platform, GPL2986 ABI Human Genome Survey Microarray version 2, Applied Biosystems; Thermo Fisher Scientific, Inc.) were obtained from the Gene Expression Omnibus (http://www.ncbi.nlm.nih. gov/geo) database and utilized as validation sets.

Data preprocessing and DEG screening. Using a z-score algorithm (18), the expression value of each gene was normalized to a normal distribution (mean $=0$; variance $=1$ ). The samples were subsequently analyzed using the differential expression via distance synthesis (DEDS) algorithm, which may be applied to obtain differential expression levels via the distance synthesis of relevant data (19). This approach was used to screen DEGs in the recurrent samples relative to the disease-free samples.

Functional enrichment analysis. Gene ontology (GO; http://www.geneontology.org) analysis may be used to predict the potential functions of gene products (20). Using GO Term Finder (http://search.cpan.org/dist/GO-TermFinder/) as previously described (21), upregulated and downregulated genes were separately enriched. Functional terms with $\mathrm{P}<0.05$ and an association with at least three genes were selected as significant terms.

Weighted gene coexpression network analysis (WGCNA). Genes may jointly influence alterations in functional terms through their interactions, with functional consistencies between genes also confirmed by significant expression level correlations. To systematically analyze how DEGs with similar expression profiles co-affect OC prognosis, WGCNA (22) was performed. Based on the obtained weighted gene coexpression levels, DEG coexpression was suggested to be significantly associated with OC prognosis. To further identify the genes that were able to differentiate between patients with OC with different prognoses, the verified DEG protein-protein interaction (PPI) pairs were used to construct a PPI network using BioNet 1.24.1 package (http://www.bioconductor. org/packages/release/bioc/html/BioNet.html).

Subnetwork analysis and classifier construction. The BioNet package (http://bionet.bioapps.biozentrum.uni-wuerzburg.de) provides an extensive framework that enables functional subnetworks to be isolated from biological networks. Using the BioNet package in R 3.1.0 (23), as previously described (24), subnetwork analysis was conducted for the PPI network with the P-value/false discovery rate set to 0.01 . With the subnetwork nodes as feature genes, a random forest classifier (25) was constructed. For sample labels (recurrence/no recurrence), true and false positive rates were calculated and combined with leave-one-out cross validation (26). Additionally, a receiver operating characteristic (ROC) curve was constructed (27) to evaluate the classification efficiency of the random forest classifier.

Validation using other independent datasets. To confirm that the subnetwork nodes were able to effectively differentiate between patients with $\mathrm{OC}$ with different prognoses, the classification efficiency of the random forest classifier for the validation set (GSE44104) was analyzed and presented using a confusion matrix. Additionally, a Kaplan-Meier (KM) survival analysis (28) was performed and combined with the clinical information belonging to the TCGA dataset.

\section{Results}

DEG screening. Following implementation of the DEDS algorithm, a total of 44 upregulated and 117 downregulated genes were identified in the recurrent samples relative to the non-recurrent samples, with more downregulated genes identified compared with upregulated genes. Additionally, a volcano plot was constructed to examine DEG expression distributions (Fig. 1).

Functional enrichment analysis. Using the GO Term Finder, significant GO terms were enriched for the upregulated and downregulated genes separately. For the upregulated genes, the enriched GO terms were primarily associated with the 'regulation of synapse assembly' $\left(\mathrm{P}=1.04 \times 10^{-7}\right)$, 'regulation of synapse organization' $\left(\mathrm{P}=5.26 \times 10^{-7}\right)$, and 'regulation of synapse structure or activity' $\left(\mathrm{P}=5.71 \times 10^{-7}\right.$; Table IA). The downregulated genes were associated with 'single-multicellular organism process' $\left(\mathrm{P}=4.11 \times 10^{-5}\right)$, 'multicellular organismal process' $\left(\mathrm{P}=5.14 \times 10^{-5}\right)$ and 'positive regulation of steroid hormone biosynthetic process' ( $\mathrm{P}=3.83 \times 10^{-3}$; Table IB), which included cytochrome $\mathrm{P} 450$ family 17 subfamily A member 1 (CYP17A1). 


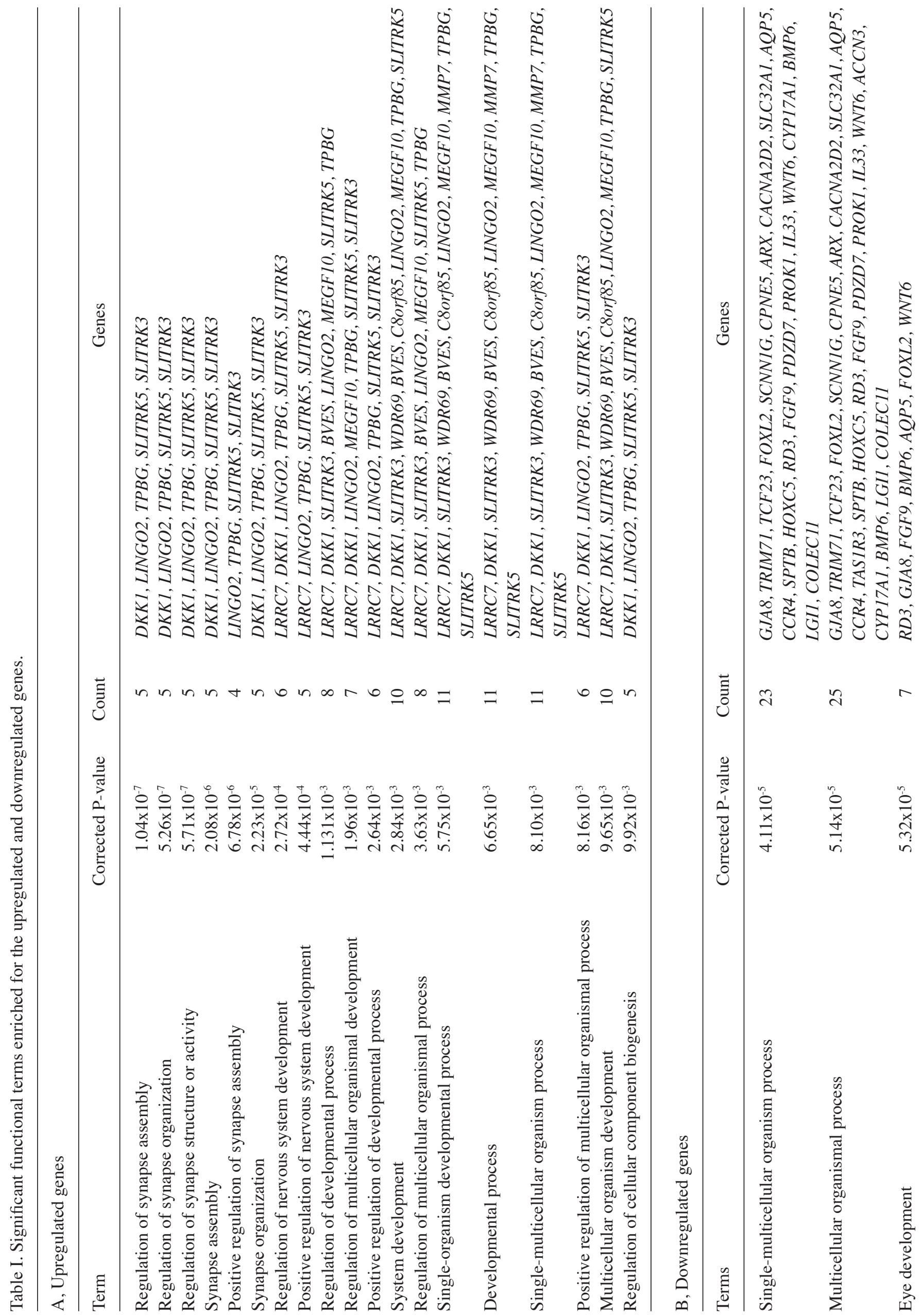




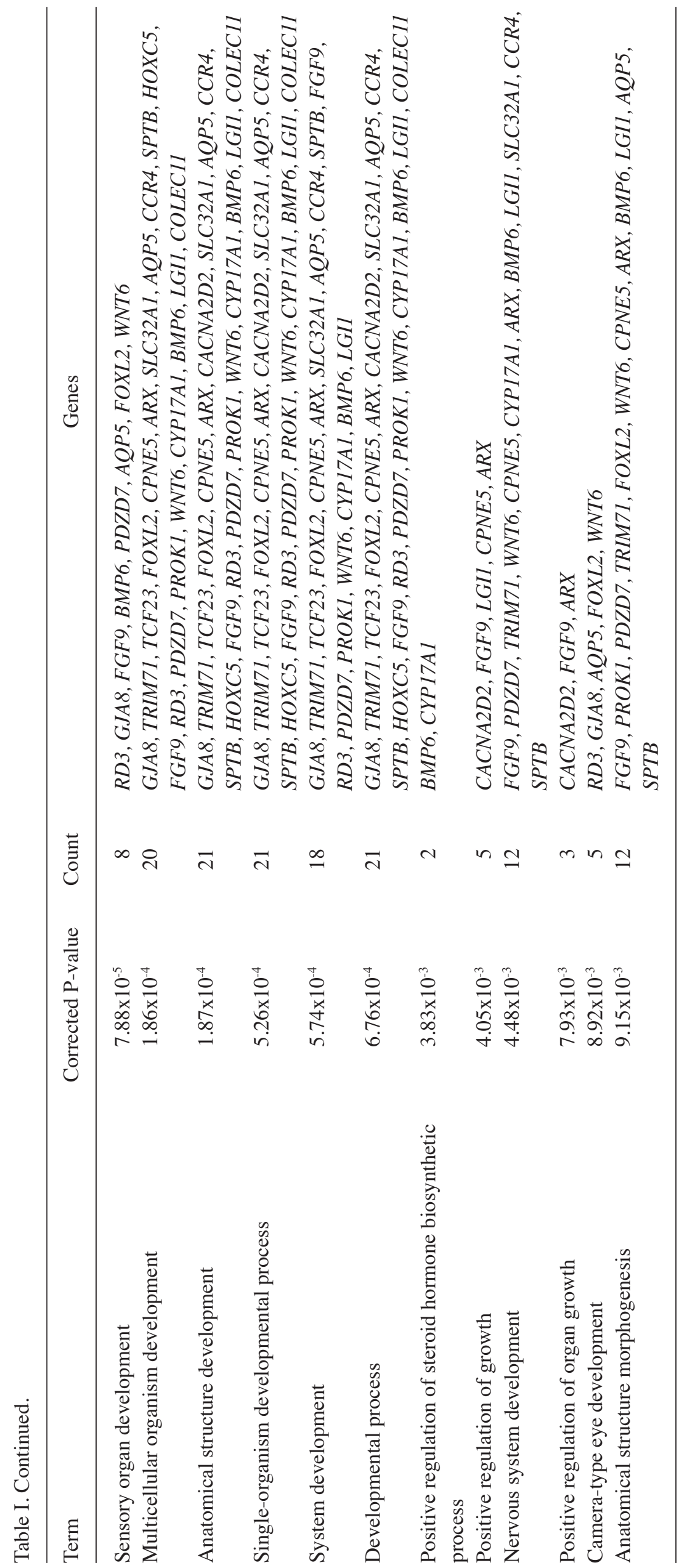




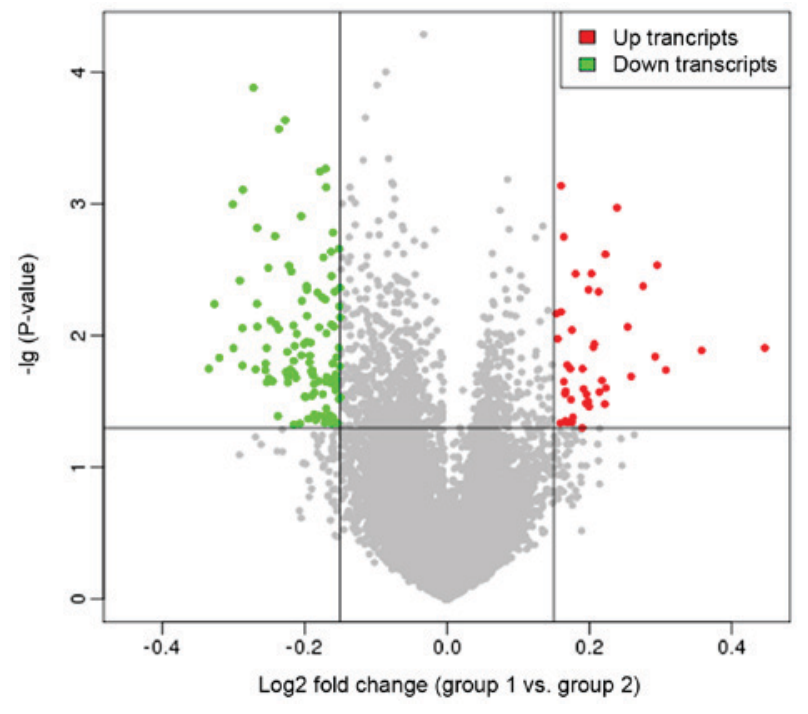

Figure 1. Volcano plot illustrating differentially expressed gene expression distributions. Upregulated genes are depicted in red and downregulated in green.

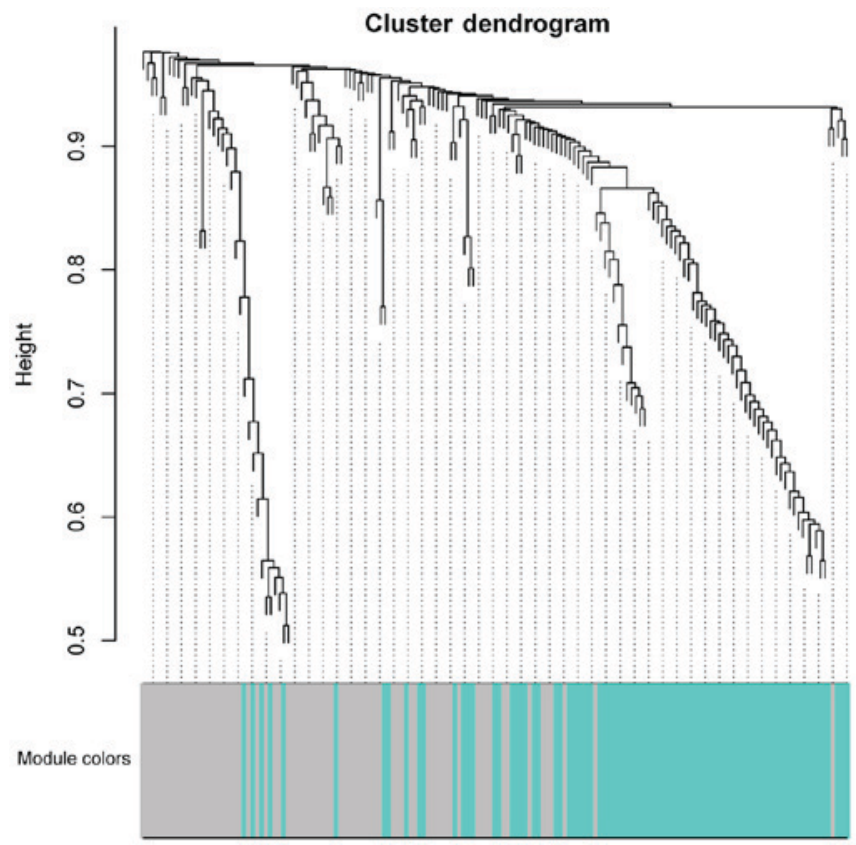

Figure 2. Weighted gene coexpression network analysis cluster dendrogram. Grey means grey module identified in WGCNA with 30 upregulated and 44 downregulated; turquoise means turquoise module identified in WGCNA with 14 upregulated and 73 downregulated genes. WGCNA, Weighted gene coexpression network analysis.

WGCNA. WGCNA was performed and turquoise and grey modules were identified within the cluster dendrogram (Fig. 2). Within the turquoise module, 87 genes were identified (14 upregulated and 73 downregulated), while in the grey module, 74 were identified (30 upregulated and 44 downregulated). Correlations between the two modules regarding status/prognosis were also analyzed. The results demonstrated that the two modules had significant correlations with status and prognosis $(\mathrm{P}<0.05$; Fig. 3). Furthermore, sample correlations were further examined via heatmap analysis, and it was indicated that the samples in the turquoise module were more

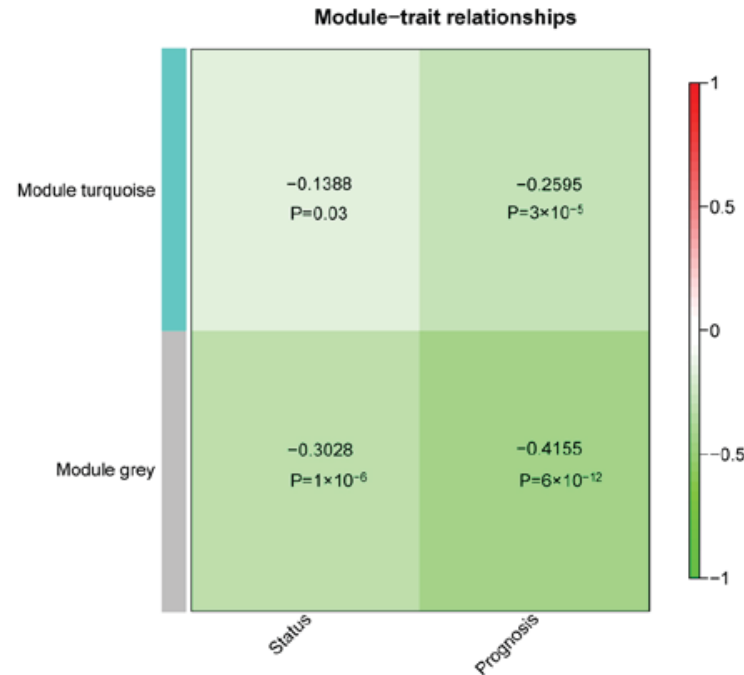

Figure 3. Module-trait relationship graph illustrating that turquoise and grey modules have significant correlations with status/prognosis. Red color indicates a positive correlation, while green means a negative correlation. Grey means grey module identified in WGCNA with 30 upregulated and 44 downregulated; turquoise means turquoise module identified in WGCNA with 14 upregulated and 73 downregulated genes.
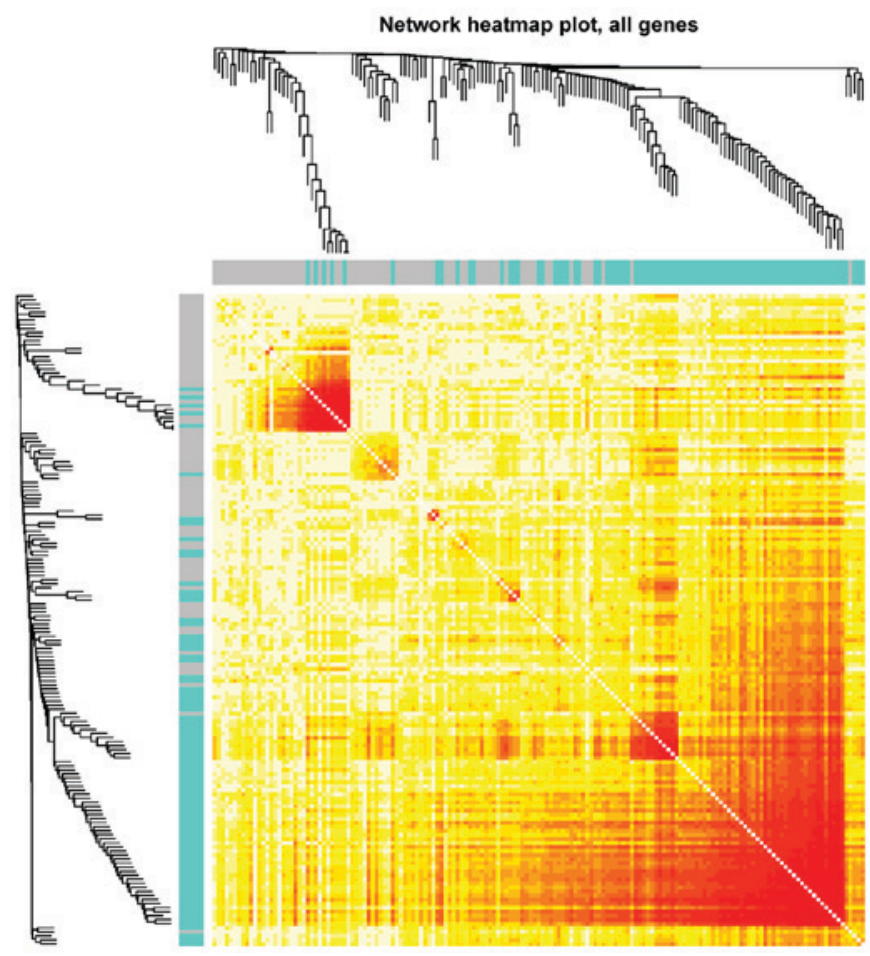

Figure 4. Heatmap displaying gene correlations for the turquoise and grey modules. A deeper color indicates a stronger correlation, while a lighter color suggests a weaker correlation. Grey means grey module identified in WGCNA; turquoise means turquoise module identified in WGCNA. WGCNA, Weighted gene coexpression network analysis.

strongly correlated when compared with those in the grey module (Fig. 4).

Subnetwork analysis and classifier construction. Following building of the PPI network, subnetwork analysis was performed and a significant subnetwork was identified (Fig. 5). The importance scores for the 16 subnetwork nodes [transcription factor 


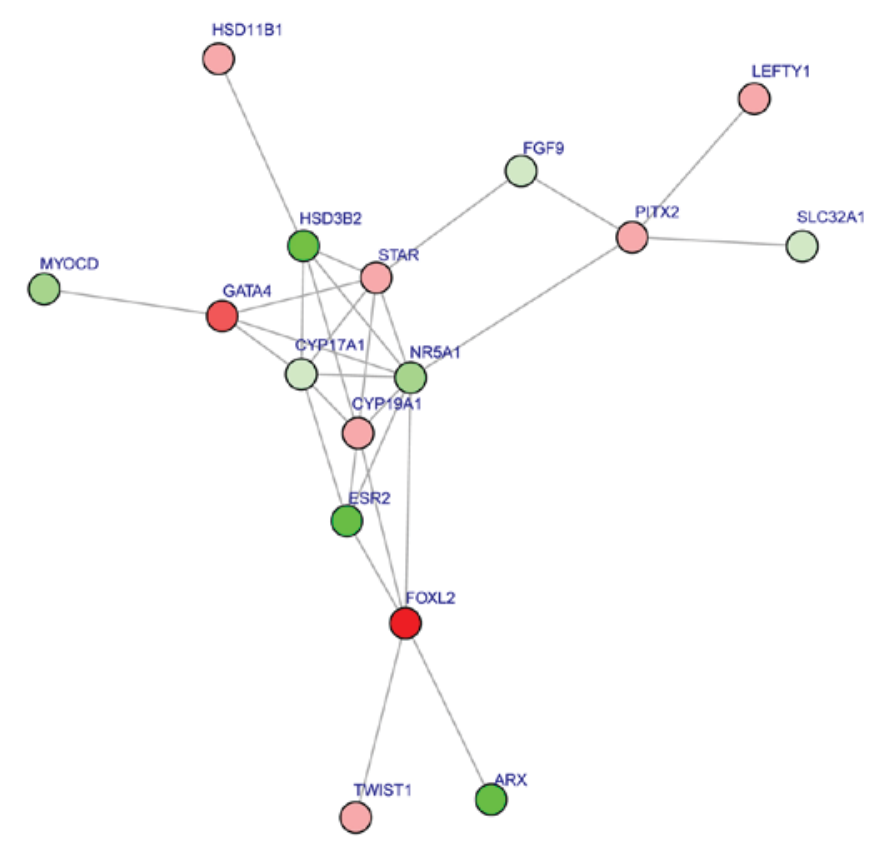

Figure 5. Significant subnetwork identified within the protein-protein interaction network. Upregulated genes are denoted in red and downregulated in green. GATA4, transcription factor GATA-4; FGF9, fibroblast growth factor 9; CYP19A1, aromatase; HSD3B2, 3 $\beta$-hydroxysteroid dehydrogenase/85-4-isomerase type 2; HSD11B1, corticosteroid 11 $\beta$-dehydrogenase isozyme 1; CYP17A1, cytochrome P450 family 17 subfamily A member 1 PITX2, pituitary homeobox 2; LEFTY1, left-right determination factor 1; ARX, homeobox protein ARX; ESR2, estrogen receptor $\beta$; NR5A1, steroidogenic factor 1; FOXL2, forkhead box protein L2; MYOCD, myocardin; STAR, steroidogenic acute regulatory protein mitochondrial; SLC32A1, vesicular inhibitory amino acid transporter; TWIST1, twist-related protein 1.

GATA-4 (GATA4); fibroblast growth factor 9 (FGF9); aromatase (CYP19A1); 33-hydroxysteroid dehydrogenase/85-4-isomerase type 2 (HSD3B2); corticosteroid 11/-dehydrogenase isozyme 1 (HSD11B1); CYP17A1; pituitary homeobox 2 (PITX2); left-right determination factor 1 (LEFTY1); homeobox protein ARX (ARX); estrogen receptor $\beta$ (ESR2); steroidogenic factor 1 (NR5A1); forkhead box protein L2 (FOXL2); myocardin (MYOCD); steroidogenic acute regulatory protein mitochondrial (STAR); vesicular inhibitory amino acid transporter (SLC32A1); and twist-related protein 1 (TWIST1)] are listed in Table II. There were multiple interactions among these subnetwork nodes, including HSD3B2-NR5A1, HSD11B1-HSD3B2, CYP17A1-GATA4, ARX-FOXL2, MYOCD-GATA4, STAR-FGF9 and SLC32A1-PITX2. The subnetwork nodes were taken as feature genes and a random forest classifier was constructed. The generated ROC curve demonstrated that the true and false positive rates separately were 92 and $23 \%$ when classifying the recurrent and non-recurrent $\mathrm{OC}$ samples (Fig. 6A).

Validation using other independent datasets. The random forest classifier was used to differentiate between samples in the GSE44104 validation set, and the prediction accuracies for the non-recurrence and recurrence groups were 87.5 and $85 \%$, respectively (Fig. $6 \mathrm{~B}$ ). These findings indicated that the subnetwork nodes were important in predicting OC prognosis.

Of the 307 OC samples in the TCGA dataset, only 262 samples remained upon removal of samples without follow-up or survival time information. These 262 samples
Table II. Importance scores of the subnetwork nodes.

\begin{tabular}{lr}
\hline Node & Score \\
\hline ARX & 15.203 \\
CYP17A1 & 10.956 \\
CYP19A1 & 10.902 \\
ESR2 & 10.537 \\
FGF9 & 10.148 \\
FOXL2 & 9.827 \\
GATA4 & 9.786 \\
HSD11B1 & 9.223 \\
HSD3B2 & 9.196 \\
LEFTY1 & 8.259 \\
MYOCD & 7.867 \\
NR5A1 & 7.634 \\
SLC32A1 & 6.367 \\
STAR & 6.167 \\
PITX2 & 5.094 \\
TWIST1 & 4.708 \\
\hline
\end{tabular}

GATA4, transcription factor GATA-4; FGF9, fibroblast growth factor 9; CYP19A1, aromatase; HSD3B2, 3 $\beta$-hydroxysteroid dehydrogenase/ 85-4-isomerase type 2; HSD11B1, corticosteroid 11 $\beta$-dehydrogenase isozyme 1; CYP17A1, cytochrome P450 family 17 subfamily A member 1; PITX2, pituitary homeobox 2; LEFTY1, left-right determination factor 1; ARX, homeobox protein ARX; ESR2, estrogen receptor $\beta$; NR5A1, steroidogenic factor 1; FOXL2, forkhead box protein L2; MYOCD, myocardin; STAR, steroidogenic acute regulatory protein mitochondrial; SLC32A1, vesicular inhibitory amino acid transporter; TWIST1, twist-related protein 1.

were divided into high- and low-risk groups using the random forest classifier, and further examined using KM survival analysis. The results demonstrated that the low risk group had a significantly longer survival time compared with the high-risk group ( $\mathrm{P}=0.0166$; Fig. 7A). Subsequently, the samples from the second validation set (GSE49997) were divided into high- and low-risk groups using the random forest classifier. Following analysis using a KM survival curve, a significant difference was noted in survival time between the low- and high-risk groups ( $\mathrm{P}=0.0165$; Fig. 7B). These findings suggested that the random forest classifier had portability and repeatability.

\section{Discussion}

In the present study, 44 upregulated and 117 downregulated genes were identified in the recurrent samples relative to the non-recurrent samples. When performing WGCNA, turquoise and grey modules were identified that had significant correlations with status and prognosis. Furthermore, a significant subnetwork was identified from the PPI network, with the subnetwork nodes (including GATA4, FGF9, CYP19A1, HSD3B2, HSD11B1, CYP17A1, PITX2, LEFTY1, ARX, ESR2, NR5A1, FOXL2, MYOCD, STAR, SLC32A1 and TWIST1) being utilized as feature genes for constructing a random forest classifier. Moreover, the classification efficiency of the random forest classifier was validated and confirmed. 
A

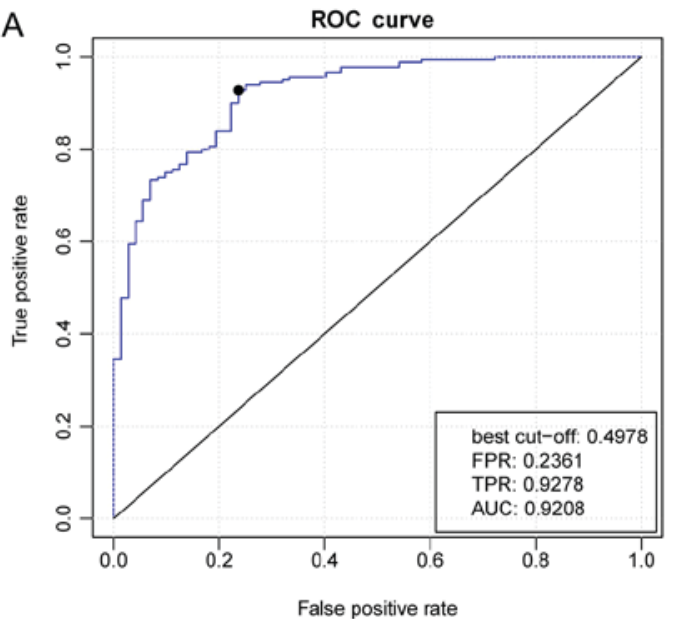

B

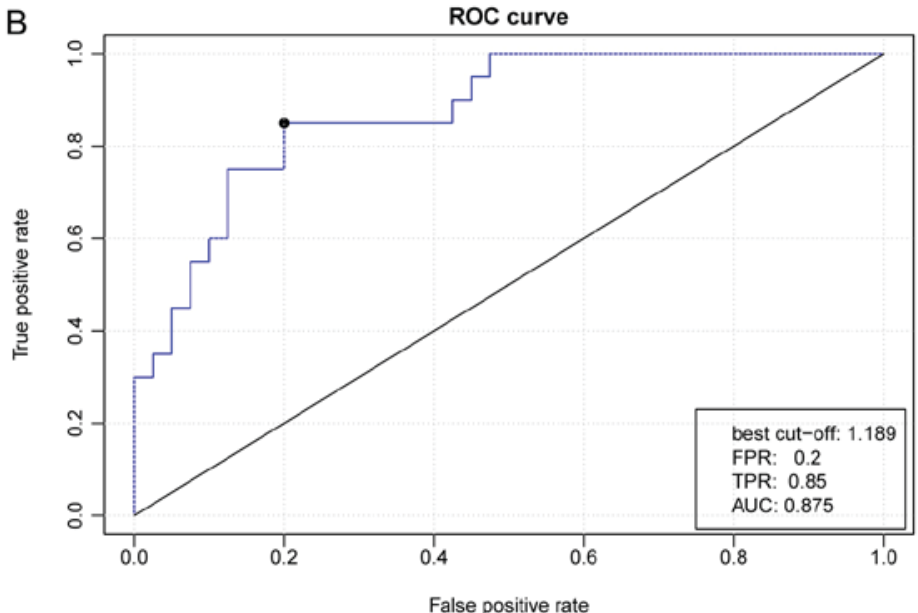

Figure 6. ROC curve analysis. ROC curves for the (A) Cancer Genome Atlas and (B) GSE44104 datasets. FPR, false positive rate; TPR, true positive rate; AUC, area under the curve; ROC, receiver operating characteristic.
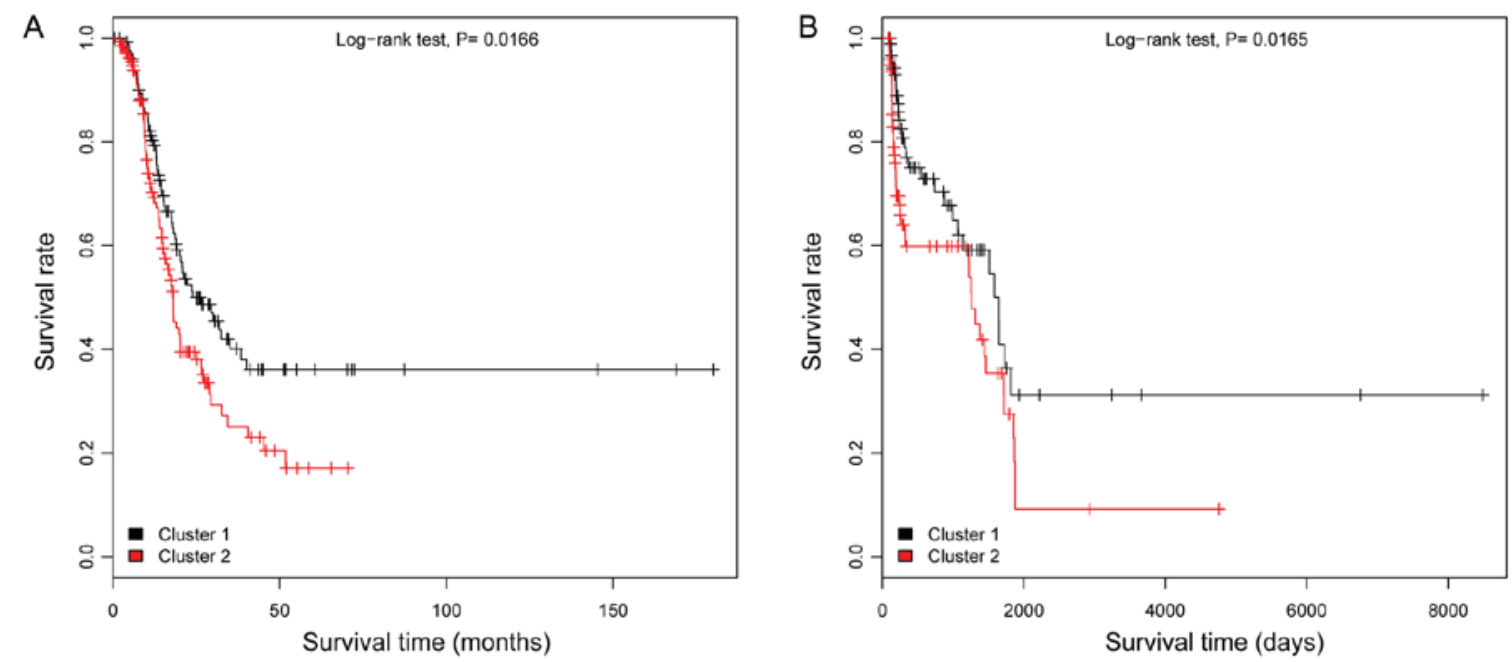

Figure 7. KM survival analysis. KM survival curves for the (A) Cancer Genome Atlas and (B) GSE49997 datasets. Predicted high risk (cluster 1) groups are indicated in red and low risk (cluster 2) groups in black. KM, Kaplan-Meier.

Of the identified nodes, previous studies have reported that GATA4 overexpression, in conjunction with human epidermal growth factor receptor 2, may predict a shorter disease-free survival time and may be utilized as a prognostic marker in patients with ovarian granulosa cell tumor to optimize follow-up management in the early stages $(29,30)$. In OC, GATA4 and transcription factor GATA-6 expression is frequently lost, with this feature specifying the histological subtype prior to tumorigenic transition of the ovarian surface epithelium (31). The upregulation of $F G F 9$ has been detected in primary ovarian endometrioid adenocarcinomas carrying a defective Wnt/ $\beta$-catenin pathway and serves an important role in promoting the cancer phenotype $(32,33)$. The aromatase enzyme encoded by the CYP19A1 gene acts in the conversion of androgen to estrogen, with CYP19A1 variants potentially able to influence OC susceptibility (34). Thus, GATA4, FGF9 and CYP19A1 appear to be implicated in the mechanisms of OC pathogenesis. Enrichment analysis demonstrated that CYP17A1, which is involved in 'positive regulation of steroid hormone biosynthetic processes', was downregulated. A previous study demonstrated that steroid hormones serve a role in $\mathrm{OC}$ pathogenesis, and their receptors influence OC patient survival (35). Therefore, CYP17Al may affect $\mathrm{OC}$ patient prognoses through the positive regulation of steroid hormones.

The overexpression of PITX2 has been implicated in OC progression by facilitating cell growth, migration and invasion, and thus may potentially serve as a therapeutic target for patients with high-grade OC $(36,37)$. PITX2 has also been demonstrated to promote OC cell proliferation through the Wnt pathway, which is closely associated with ovarian development and OC (38). In ovarian clear cell carcinoma, the overexpression of LEFTY, a transforming growth factor- $\beta$ superfamily member, exhibits an anti-tumor effect by affecting cell proliferation and cellular susceptibility to apoptotic signals (39). Estrogen receptor $\beta$, which is encoded by the ESR 2 gene, has been suggested to serve as a critical factor during OC carcinogenesis (40). Furthermore, within the ESR 2 promoter region, the genotypic and allelic frequencies of the single nucleotide polymorphism (SNP) rs3020449 
have been demonstrated to exhibit significant differences based on OC stage, thus indicating that SNP rs3020449 may be associated with OC progression (41). These findings suggest that PITX2, LEFTY1 and ESR2 may also serve roles in OC pathogenesis.

NR5Al serves an important role in ovarian function and development, with an NR5A1 mutation reported to induce $46 \mathrm{XY}$ disorders of sex development (42). FOXL2 is critical for GC (granulosa cell) differentiation during the process of folliculogenesis, with its downregulation potentially serving as an ovarian granulosa cells tumor prognostic factor (43). TWIST1, which may induce epithelial-mesenchymal transition and contribute to tumor metastasis, has been demonstrated to be associated with poor survival in patients with cancer $(44,45)$. Thus, NR5A1, FOXL2 and TWIST1 may be associated with patient survival in OC. Moreover, the multiple interactions within the PPI subnetwork (including HSD3B2-NR5A1, HSD11B1-HSD3B2, CYP17A1-GATA4, ARX-FOXL2, MYOCD-GATA4, STAR-FGF9 and SLC32A1-PITX2) indicate that HSD3B2, HSD11B1, CYP17A1, ARX, MYOCD, STAR and $S L C 32 A 1$ may also function in OC by interacting with other genes.

However, the present study has several limitations to note. First, the datasets used in the present study had sample size differences, platform differences and data heterogeneities that may affect the prediction accuracy of the random forest classifier. Second, the smaller patient numbers and analytical methods may limit the predictive capability of the present model. Finally, only bioinformatics analyses were conducted in the present study, and no direct experimental validation was performed. Therefore, further analyses are required to validate the obtained results.

In conclusion, 44 upregulated and 117 downregulated genes associated with OC recurrence were identified. Furthermore, the 16 subnetwork node genes that were identified may be critical molecules associated with $\mathrm{OC}$ recurrence.

\section{Acknowledgements}

Not applicable.

\section{Funding}

The present study was supported by grants from the Hubei Province's Outstanding Medical Academic Leader Program and the Hubei Province Health and Family Planning Scientific Research Project (grant no. WJ2015MA024) and the Natural Science Foundation of Hubei Province (grant no. 2017CFB335).

\section{Availability of data and materials}

The datasets used and/or analysed during the current study are available from the corresponding author on reasonable request.

\section{Authors' contributions}

LC and LL performed data analyses and wrote the manuscript. LW, XL and HX contributed significantly in data analyses and manuscript revision. JZ conceived and designed the study. All authors read and approved the final manuscript.

\section{Ethics approval and consent to participate}

Not applicable.

\section{Consent for publication}

Not applicable.

\section{Competing interests}

The authors declare that they have no competing interests.

\section{References}

1. Jayson GC, Kohn EC, Kitchener HC and Ledermann JA: Ovarian cancer. Lancet 384: 1376-1388, 2014.

2. Ebell MH, Culp MB and Radke TJ: A systematic review of symptoms for the diagnosis of ovarian cancer. Am J Prev Med 50: 384-394, 2016.

3. McGuire S: World Cancer Report 2014. Geneva, Switzerland: World Health Organization, International Agency for Research on Cancer, WHO Press, 2015. Adv Nutr 7: 418-419, 2016.

4. Lengyel E: ovarian cancer development and metastasis. Am J Pathol 177: 1053-1064, 2010.

5. Cree IA: Cancer biology. Methods Mol Biol 731: 1-11, 2011

6. Holschneider CH and Berek JS: Ovarian cancer: Epidemiology, biology, and prognostic factors. Semin Surg Oncol 19: 3-10, 2000.

7. Tworoger SS and Doherty JA: Epidemiologic paradigms for progress in ovarian cancer research. Cancer Causes Control 28: 361-364, 2017.

8. Siu MK, Chan HY, Kong DS, Wong ES, Wong OG, Ngan HY, Tam KF, Zhang H, Li Z, Chan QK, et al: p21-activated kinase 4 regulates ovarian cancer cell proliferation, migration, and invasion and contributes to poor prognosis in patients. Proc Natl Acad Sci USA 107: 18622-18627, 2010.

9. Nakayama N, Nakayama K, Shamima Y, Ishikawa M, Katagiri A, Iida K and Miyazaki K: Gene amplification CCNE1 is related to poor survival and potential therapeutic target in ovarian cancer. Cancer 116: 2621-2634, 2010.

10. Etemadmoghadam D, George J, Cowin PA, Cullinane C, Kansara M; Australian Ovarian Cancer Study Group, Gorringe KL, Smyth GK and Bowtell DD: Amplicon-dependent CCNE1 expression is critical for clonogenic survival after cisplatin treatment and is correlated with 20q11 gain in ovarian cancer. PLoS One 5: e15498, 2010.

11. Ehlén Å, Brennan DJ, Nodin B, O'Connor DP, Eberhard J, Alvarado-Kristensson M, Jeffrey IB, Manjer J, Brändstedt J, Uhlén M, et al: Expression of the RNA-binding protein RBM3 is associated with a favourable prognosis and cisplatin sensitivity in epithelial ovarian cancer. J Transl Med 8: 78, 2010.

12. Xia Y, Chang T, Wang Y, Liu Y, Li W, Li M and Fan HY: YAP promotes ovarian cancer cell tumorigenesis and is indicative of a poor prognosis for ovarian cancer patients. PLoS One 9: e91770, 2014.

13. Zhang X, George J, Deb S, Degoutin JL, Takano EA, Fox SB; AOCS Study group, Bowtell DD and Harvey KF: The Hippo pathway transcriptional co-activator, YAP, is an ovarian cancer oncogene. Oncogene 30: 2810-2822, 2011.

14. Zhang J, Guo X, Chang DY, Rosen DG, Mercado-Uribe I and Liu J: CD133 expression associated with poor prognosis in ovarian cancer. Mod Pathol 25: 456-464, 2012.

15. Servant N, Roméjon J, Gestraud P, La Rosa P, Lucotte G, Lair S, Bernard V, Zeitouni B, Coffin F, Jules-Clément G, et al: Bioinformatics for precision medicine in oncology: Principles and application to the SHIVA clinical trial. Front Genet 5: 152, 2014.

16. Altshuler D, Daly MJ and Lander ES: Genetic mapping in human disease. Science 322: 881-888, 2008.

17. Chen JM, Cooper DN, Chuzhanova N, Férec C and Patrinos GP: Gene conversion: Mechanisms, evolution and human disease. Nat Rev Genet 8: 762-775, 2007.

18. Segundo ES, Tsanas A and Gómez-Vilda P: Euclidean distances as measures of speaker dissimilarity including identical twin pairs: A forensic investigation using source and filter voice characteristics. Forensic Sci Int 270: 25-38, 2017. 
19. Yang YH, Xiao Y and Segal MR: Identifying differentially expressed genes from microarray experiments via statistic synthesis. Bioinformatics 21: 1084-1093, 2005.

20. Tweedie S, Ashburner M, Falls K, Leyland P, McQuilton P, Marygold S, Millburn G, Osumi-Sutherland D, Schroeder A, Seal R, et al: FlyBase: Enhancing Drosophila gene ontology annotations. Nucleic Acids Res 37 (Database issue): D555-D559, 2009.

21. Lässer C, O'Neil SE, Shelke GV, Sihlbom C, Hansson SF, Gho YS, Lundbäck B and Lötvall J: Exosomes in the nose induce immune cell trafficking and harbour an altered protein cargo in chronic airway inflammation. J Transl Med 14: 181, 2016.

22. Iancu OD, Colville A, Oberbeck D, Darakjian P, Mcweeney SK and Hitzemann R: Cosplicing network analysis of mammalian brain RNA-Seq data utilizing WGCNA and Mantel correlations. Front Genet 6: 174, 2015.

23. RC Team: R: A language and environment for statistical computing. R Foundation for Statistical Computing, Vienna, Austria, 2016.

24. Beisser D, Klau GW, Dandekar T, Müller T and Dittrich MT: BioNet: An R-Package for the functional analysis of biological networks. Bioinformatics 26: 1129-1130, 2010.

25. Ma X, Guo J and Sun X: DNABP: Identification of DNA-binding proteins based on feature selection using a random forest and predicting binding residues. PLoS One 11: e0167345, 2016.

26. Mikshowsky A, Weigel KA and Gianola D: 0294 Assessing genomic prediction accuracy for Holstein sires using bootstrap aggregation sampling and leave-one-out cross validation. J Anim Sci 94: 139-140, 2016.

27. Thomas M: ROC curve comparison of ICNARC and POSSUM in a UK critical care unit. Br J Anaesth 117: 667, 2016.

28. May WL: Kaplan-Meier survival analysis. In: Encyclopedia of Cancer. Schwab M (ed) Springer, Berlin, pp1934-1937, 2011.

29. Färkkilä A, Andersson N, Bützow R, Leminen A, Heikinheimo M Anttonen M and Unkila-Kallio L: HER2 and GATA4 are new prognostic factors for early-stage ovarian granulosa cell tumor-a long-term follow-up study. Cancer Med 3: 526-536, 2014.

30. Anttonen M, Pihlajoki M, Andersson N, Georges A, L'hôte D, Vattulainen S, Färkkilä A1, Unkila-Kallio L, Veitia RA and Heikinheimo M: FOXL2, GATA4, and SMAD3 co-operatively modulate gene expression, cell viability and apoptosis in ovarian granulosa cell tumor cells. PLoS One 9: e85545, 2014.

31. Cai KQ, Caslini C, Capo-Chichi CD, Slater C, Smith ER, Wu H, Klein-Szanto AJ, Godwin AK and Xu XX: Loss of GATA4 and GATA6 expression specifies ovarian cancer histological subtypes and precedes neoplastic transformation of ovarian surface epithelia. PLoS One 4: e6454, 2009.

32. Hendrix ND, Wu R, Kuick R, Schwartz DR, Fearon ER and Cho KR: Fibroblast growth factor 9 has oncogenic activity and is a downstream target of Wnt signaling in ovarian endometrioid adenocarcinomas. Cancer Res 66: 1354-1362, 2006.

33. Drummond AE, Tellbach M, Dyson $M$ and Findlay JK: Fibroblast growth factor-9, a local regulator of ovarian function. Endocrinology 148: 3711-3721, 2007.
34. Goodman MT, Lurie G, Thompson PJ, Mcduffie KE and Carney ME: Association of two common single-nucleotide polymorphisms in the CYP19A1 locus and ovarian cancer risk. Endocr Relat Cancer 15: 1055-1060, 2008.

35. Jönsson JM, Arildsen NS, Malander S, Måsbäck A, Hartman L, Nilbert $\mathrm{M}$ and Hedenfalk I: Sex steroid hormone receptor expression affects ovarian cancer survival. Transl Oncol 8: 424-433, 2015.

36. Fung FK, Chan DW, Liu VW, Leung TH, Cheung AN and Ngan HY: Increased expression of PITX2 transcription factor contributes to ovarian cancer progression. PLoS One 7: e37076, 2012.

37. Basu M, Bhattacharya R, Ray U, Mukhopadhyay S, Chatterjee U and Roy SS: Invasion of ovarian cancer cells is induced byPITX 2-mediated activation of TGF- $\beta$ and Activin-A. Mol Cancer 14: 162, 2015.

38. Basu M and Roy SS: Wnt/ $\beta$-catenin pathway is regulated by PITX2 homeodomain protein and thus contributes to the proliferation of human ovarian adenocarcinoma cell, SKOV-3. J Biol Chem 288: 4355-4367, 2013.

39. Akiya M, Yamazaki M, Matsumoto T, Kawashima Y, Oguri Y, Kajita S, Kijima D, Chiba R, Yokoi A, Takahashi H, et al: Identification of LEFTY as a molecular marker for ovarian clear cell carcinoma. Oncotarget 8: 63646-63664, 2017.

40. Leigh Pearce C, Near AM, Butler JL, Van Den Berg D, Bretsky P, Conti DV, Stram DO, Pike MC, Hirschhorn JN and Wu AH: Comprehensive evaluation of ESR2 variation and ovarian cancer risk. Cancer Epidemiol Biomarkers Prev 17: 393-396, 2008.

41. Schüler S, Lattrich C, Skrzypczak M, Fehm T, Ortmann O and Treeck O: Polymorphisms in the promoter region of ESR2 gene and susceptibility to ovarian cancer. Gene 546: 283-287, 2014.

42. Lourenço D, Brauner R, Lin L, De Perdigo A, Weryha G, Muresan M, Boudjenah R, Guerra-Junior G, Maciel-Guerra AT, Achermann JC, et al: Mutations in NR5A1 Associated with Ovarian Insufficiency. N Engl J Med 360: 1200-1210, 2009.

43. Kalfa N, Philibert P, Patte C, Ecochard A, Duvillard P, Baldet P, Jaubert F, Fellous M and Sultan C: Extinction of FOXL2 expression in aggressive ovarian granulosa cell tumors in children. Fertil Steril 87: 896-901, 2007.

44. Wushou A, Hou J, Zhao YJ and Shao ZM: Twist-1 up-regulation in carcinoma correlates to poor survival. Int J Mol Sci 15: 21621-21630, 2014

45. Yin G, Alvero AB, Craveiro V, Holmberg JC, Fu HH, Montagna MK, Yang Y, Chefetz-Menaker I, Nuti S, Rossi M, et al: Constitutive proteasomal degradation of TWIST-1 in epithelial-ovarian cancer stem cells impacts differentiation and metastatic potential. Oncogene 32: 39-49, 2013.

(c) (i) () $९$ This work is licensed under a Creative Commons cc) $\mathrm{EY}$ No No Attribution-NonCommercial-NoDerivatives 4.0 International (CC BY-NC-ND 4.0) License. 